\title{
Carteira de Serviços da Atenção Primária à Saúde: garantia de integralidade nas Equipes de Saúde da Família e Saúde Bucal no Brasil
}

\author{
Primary Health Care Portfolio: Assuring of integrality in the Family \\ Health and Oral Health Teams in Brazil
}

Carlo Roberto Hackmann da Cunha (https://orcid.org/0000-0002-3545-5801) ${ }^{1}$

Erno Harzheim (https://orcid.org/0000-0002-8919-7916) ${ }^{2}$

Olivia Lucena de Medeiros (https://orcid.org/0000-0002-4459-6665) ${ }^{2}$

Otávio Pereira D’Avila (https://orcid.org/0000-0003-1852-7858) ${ }^{2}$

Caroline Martins (https://orcid.org/0000-0003-3384-2637) ${ }^{2}$

Lucas Wollmann (https://orcid.org/0000-0002-3543-0794) ${ }^{2}$

Lívia de Almeida Faller (https://orcid.org/0000-0001-9905-0507) ${ }^{2}$

${ }^{1}$ Departamento de Ciências Biológicas e da Saúde e de Ciências Sociais Aplicadas, Universidade do Sul de Santa Catarina. Av. José Acácio Moreira 787, Bairro Dehon. 88704-900 Tubarão SC Brasil.

carlorhc@gmail.com

${ }^{2}$ Secretaria de Atenção Primária à Saúde,

Ministério da Saúde. Brasília DF Brasil.

\begin{abstract}
More than 30 years into the anniversary of the Unified Health System (SUS), 40 years after Alma-Ata, and soon after the Astana Conference, the Brazilian Ministry of Health proposes several strategies to strengthen PHC with the creation of the Primary Health Care Secretariat (SAPS). This paper presents the process of developing the national PHC service portfolio, one of the strategies developed by SAPS to strengthen the PHC clinic, and the challenges for the expansion of comprehensive care in the actions developed by the Family Health and Oral Health teams. After the public consultation, from a total of 209 initially listed actions and procedures, including incorporations and exclusions, 210 items were defined, including the actions planned for the integration between primary care and health surveillance. We emphasize that the national portfolio model can be adapted to the reality and municipal context in each of the federation units, including considering the availability of the local care network.
\end{abstract} Key words Primary health care, Comprehensiveness, Family health, Oral health, Brazil
Resumo Ao ultrapassarmos os 30 anos do Sistema Único de Saúde (SUS), após 40 anos de Alma-Ata e logo após a Conferência de Astana, o Ministério da Saúde do Brasil propõe diversas estratégias de fortalecimento da APS com a criação da Secretaria de Atenção Primária à Saúde (SAPS). Este artigo apresenta o processo de desenvolvimento da carteira nacional de serviços para a APS, uma das estratégias desenvolvidas pela SAPS para fortalecimento da clínica na APS, e os desafios para a ampliação da integralidade do cuidado nas ações desenvolvidas pelas equipes de Saúde da Família e Saúde Bucal. Após a consulta pública, de um total de 209 ações e procedimentos inicialmente listados, entre incorporações e exclusões, foram definidos 210 itens, incluindo as ações previstas para a integração entre atenção primária e vigilância em saúde. Ressaltamos que o modelo da carteira nacional pode ser adaptado à realidade $e$ contexto municipal em cada uma das unidades da federação, inclusive considerando a disponibilidade da rede de atenção local.

Palavras-chave Atenção primária à saúde, Integralidade, Saúde da família, Saúde bucal, Brasil 


\section{Introdução}

Em agosto de 2007, a Organização Pan-Americana da Saúde (OPAS) divulgou que a principal abordagem para produzir melhoras sustentáveis e equitativas na saúde das populações das Américas seria por meio do desenvolvimento de sistemas de saúde fortemente embasados na Atenção Primária à Saúde $(\mathrm{APS})^{1}$. Em 2008, a OMS, em seu Relatório Mundial de Saúde, reforçou esta orientação com a afirmação que dá nome àquela publicação: APS - agora mais do que nunca! ${ }^{2}$ Mais recentemente, em outubro de 2018, ao completarmos 40 anos de Alma-Ata, a Conferência Global sobre APS produziu o documento intitulado Declaração de Astana que salienta ser a APS "o enfoque mais eficaz, eficiente e equitativo para melhorar a saúde, o que faz dela um alicerce necessário para conseguir a cobertura universal de saúde"s.

Ao completarmos 30 anos do SUS, após 40 anos de Alma-Ata ${ }^{4}$ e logo após a Conferência de Astana $^{3}$, o Ministério da Saúde do Brasil propõe diversas estratégias de fortalecimento da APS com a criação da Secretaria de Atenção Primária à (SAPS $)^{5}$. Como competências e compromissos da SAPS, definidas pelo decreto mencionado, destacam-se: ampliar o acesso da população às unidades de saúde da família, definir um novo modelo de financiamento baseado em resultados em saúde e eficiência, estabelecer um novo modelo de provimento e formação de médicos para áreas remotas, fortalecer a clínica da APS e o trabalho em equipe e ampliar a informatização das unidades e a utilização de prontuário eletrônico.

O fortalecimento da clínica e do trabalho em equipe na Estratégia Saúde da Família (ESF) passa pelo reconhecimento dos entes federados sobre a importância da APS para a organização dos serviços. Para isso, além da infraestrutura física das Unidades de Saúde da Família (USF), há que se ter condições de trabalho e divisão clara e com subsidiariedade das atribuições de cada um dos profissionais a partir das ações e procedimentos ofertados à população, sem prejuízo dos processos de cuidado compartilhado entre os profissionais. Nesse sentido, a criação de uma "carteira de serviços de atenção primária" poderá ajudar na definição dos papéis que cada um deverá desempenhar, além de, fundamentalmente, apresentar os procedimentos e os serviços ofertados nesse ambiente de cuidado, reduzindo a heterogeneidade de práticas existentes na APS nacional entre os municípios, mas também entre unidades de um mesmo município, para que a população brasileira possa reconhecer o que se espera de ações e serviços em uma Unidade de Saúde da Família.

Sistemas universais de saúde são sustentáveis se possuírem uma APS forte ${ }^{6,7}$. A APS objetiva melhor saúde individual e populacional com equidade. Este objetivo só é alcançado caso os serviços de APS funcionarem adequadamente, isto é, se aliarem alta resolutividade clínica com responsabilização pela saúde da população sob cuidado e pela comunicação adequada dos fatos e eventos que caracterizam a trajetória clínica das pessoas. Para atingir os objetivos acima e funcionar adequadamente é essencial que a APS seja organizada com a máxima presença e extensão de suas características operacionais, de seus atributos. Os atributos essenciais da Atenção Primária (acesso de primeiro contato; longitudinalidade; coordenação; integralidade) são características operacionais, cotidianas e mensuráveis dos serviços de $\mathrm{APS}^{8}$. Quanto mais forte a presença e a extensão desses atributos, mais forte será a APS. Para fortalecer cada um dos atributos, serão necessárias ferramentas organizacionais diferentes e complementares.

No Brasil, a carteira de serviços é uma das mais importantes ferramentas organizacionais. Já é utilizada no Rio de Janeiro, em Curitiba e Florianópolis, entre outros lugares ${ }^{9-11}$. Ela se configura como um importante instrumento para a garantia da integralidade. A APS deve organizarse de tal forma que o cidadão tenha todos os serviços de saúde necessários, identificando e proporcionando os serviços preventivos, bem como os que possibilitem o diagnóstico e o tratamento das doenças, estabelecendo também a forma adequada para resolução de problemas, sejam orgânicos, funcionais ou sociais.

Ao se definir uma lista transparente de ações e serviços voltados aos problemas e às condições de saúde mais frequentes temos a 'alavanca' necessária para mover o 'mundo' da integralidade e trazê-lo para o cotidiano da vida das pessoas e das equipes de APS. A carteira de serviços deixa claro para as pessoas quais serviços e ações elas podem encontrar nas Unidades de APS e permite aos profissionais se organizarem na rotina do atendimento, assim como buscarem conhecimentos e habilidades para ofertar as ações e os serviços com competência. Além disso, a carteira torna-se um instrumento de monitoramento da gestão, que deve prover as condições estruturais (equipamentos, insumos, recursos humanos, financiamento) e de processo de trabalho - forma de adscrição das pessoas, estratégias de desenvol- 
vimento pessoal contínuo, monitoramento e avaliação - suficientes para que suas ações e serviços sejam realidade no dia-a-dia da APS.

Destinada aos profissionais da Rede de Atenção à Saúde (RAS), contém a definição das responsabilidades da APS com vistas à garantia fundamental do atributo integralidade/abrangência do cuidado. Busca delimitar diretrizes gerais de organização e definir ações e procedimentos ofertados em cada serviço a partir de critérios essencialmente estruturais. A APS deve ser responsável pelos problemas mais comuns e frequentes de saúde da população e resolver de $80 \%$ a $90 \%$ de suas demandas. Do ponto de vista clinico, a Carteira de Serviços não visa enumerar nem esgotar todos os sinais/sintomas ou patologias mais prevalentes que devem ser manejados e acompanhados pelo cuidado na APS, especialmente porque a epidemiologia e as necessidades das pessoas são dinâmicas e variadas, principalmente quando falamos de um país com dimensões continentais como o Brasil. Ainda, vale ressaltar que os procedimentos e ações a serem realizados devem respeitar as regulamentações específicas dos conselhos profissionais, bem como as habilidades individuais, sendo mote para a organização e a identificação de treinamentos adicionais necessários.

O objetivo central da carteira de serviços é guiar os profissionais em relação aos que se espera que sejam ofertados nas unidades de APS (especialmente no contexto das diretrizes e metas da SAPS e MS) e se existe alguma condição particular para que este ocorra (tabela de ações/ programas e materiais). Além disso, serve para reforçar quais critérios são indicativos para manejar condições ou queixas comuns em um nível maior de complexidade da rede do município, em caráter eletivo (não estão contempladas situações de emergência). É um documento que não visa ser excludente, portanto, a ausência de menção de um sinal, sintoma ou doença não significa que não se deva realizar seu atendimento na APS.

O objetivo deste artigo é apresentar o desenvolvimento da carteira nacional de serviços para a APS e os desafios para o fortalecimento da integralidade do cuidado nas ações desenvolvidas pelas equipes de Saúde da Família e de Saúde Bucal.

\section{Material e métodos}

Trata-se de um estudo com análise documental, visando à produção de uma orientação técnica que possa ajudar as equipes de APS e a gestão em nível estadual e municipal na definição da oferta de ações e serviços nas unidades de saúde da família. A chamada "Carteira de Serviços da Atenção Primária à Saúde" (CASAPS) é um documento que visa nortear as ações de saúde na APS brasileira com reconhecimento da clínica multiprofissional. É um documento orientador para todos os serviços de APS no Brasil, sendo, portanto, um instrumento que visa contribuir para o fortalecimento da oferta de cuidados próprios da APS. É uma ferramenta importante para a gestão do cuidado que será revisada de forma regular pela SAPS. Ela se destina a todos os profissionais, gestores e cidadãos brasileiros para que se apropriem e tenham conhecimento dos serviços de saúde oferecidos na APS. Uma de suas versões é destinada especificamente aos profissionais e gestores, contendo a lista de serviços e insumos e equipamentos necessários (Tabela 1).

Para sua elaboração consideramos os seguintes materiais: (i) revisão de documentos nacionais e internacionais que apresentam carteiras de serviços de APS. Inicialmente, foi realizada uma ampla revisão de carteiras de serviços da APS publicadas no Brasil e no exterior. Por ser uma ferramenta pouco utilizada em nosso país, revisamos as carteiras do Rio de Janeiro, Florianópolis, Curitiba (Carteira de Serviços e Cartilha de Acesso), Belo Horizonte, Natal e Porto Alegre. Foram também revisadas as carteiras de serviços de Portugal, da Espanha e da cidade de Madrid; (ii) revisão do Manual do Instrumento de Avaliação da Atenção Primária (PCATool) publicado em 2010 pelo Ministério da Saúde ${ }^{12}$.

Foram elencados todos os serviços descritos nas carteiras de municípios citados anteriormente e realizado um comparativo entre elas, construindo-se um quadro com a listagem dos termos e verificação das cidades onde o serviço constava como presente na carteira. Os serviços foram divididos e apresentados da seguinte forma: atenção à saúde do adulto e do idoso, atenção à saúde da criança e do adolescente, procedimentos na APS e Saúde Bucal. Além disso, foi elaborado um texto de orientação com relação ao processo de trabalho das equipes de saúde na APS tendo os atributos essenciais da APS como eixos-chave.

As etapas da consulta pública foram: (i) interação com avaliadores externos para contribuição na elaboração do conteúdo da carteira; (ii) disponibilização do esboço da carteira de serviços durante o período da consulta pública no Portal do Ministério da Saúde, juntamente com um formulário na Plataforma Google Forms em agosto/2019. Neste momento, o participante ma- 
Tabela 1. Grau de concordância com a inclusão dos serviços propostos na consulta pública para a construção de uma carteira nacional de serviços para a APS.

\begin{tabular}{lccccc}
\hline \multirow{2}{*}{$\begin{array}{c}\text { Grau de } \\
\text { concordância }\end{array}$} & Adulto e Idoso & $\begin{array}{c}\text { Criança e } \\
\text { Adolescente }\end{array}$ & $\begin{array}{c}\text { Procedimentos } \\
\text { na APS }\end{array}$ & Saúde Bucal & $\begin{array}{c}\text { Carteira de } \\
\text { Serviços geral }\end{array}$ \\
\cline { 2 - 6 } Acima de 90\% & 93,0 & 94,0 & 45,0 & 78,0 & 77,6 \\
Entre 80 e 89,9\% & 3,0 & 6 & 35,0 & 22,0 & 16,6 \\
Entre 70 e 79,9\% & 2,0 & - & 20,0 & - & 5,4 \\
Abaixo de 60\% & 2,0 & - & - & - & 0,4 \\
\hline
\end{tabular}

Fonte: Elaboração dos autores, a partir da consulta pública realizada.

nifestou sua opinião pela inclusão ou exclusão e pôde sugerir alterações para cada item de lista de ações e procedimentos ${ }^{13}$ (iii) revisão após a consulta pública pela SAPS do texto inicial e da lista de ações e serviços apresentadas.

\section{A contribuição das Associações Profissionais, do Conselho Nacional de Secretários Estaduais de Saúde (CONASS) e do Conselho Nacional dos Secretários Municipais de Saúde (CONASEMS)}

Além de outras Secretarias do próprio Ministério da Saúde, ocorreu o envio da proposta para manifestação do CONASS, CONASEMS e das Associações de profissionais da Sociedade Brasileira de Medicina de Família e Comunidade (SBMFC), Associação Brasileira de Enfermagem de Família e Comunidade (ABEFACO) e Associação Brasileira de Odontologia (ABO), os quais foram chamados de avaliadores externos. Tais entidades foram consultadas tanto no período da consulta pública, quanto em momento posterior, sobre a versão final da carteira resultante da consulta pública. Nesta fase, essas instâncias também opinaram sobre a classificação em padrões essenciais e ampliados de APS previstos na PNAB.

\section{Resultados da versão final da carteira de serviços}

Após o término da consulta pública realizou-se uma análise quantitativa e qualitativa das respostas enviadas pelos formulários eletrônicos. Ao todo foram respondidos 1.855 formulários, distribuídos com base no perfil do respondente da seguinte forma: $1.415(76,3 \%)$ profissionais de saúde, 86 (4,6\%) usuários do SUS, 81 (4,4\%) estudantes, $121(6,5 \%)$ gestores municipais, 28 $(1,5 \%)$ gestores estaduais, $17(0,9 \%)$ gestores do Ministério da Saúde, 44 (2,4\%) pesquisadores e $63(3,4 \%)$ identificado na categoria “outros".

O grau de concordância (pessoas que assinalaram "concordo com a inclusão" ou "concordo com a inclusão com alterações") foi elevado (Tabela 1). O item com menor percentual de concordância $(46,7 \%)$ foi: "Identificação quanto à posse de arma de fogo por parte do adulto e orientação sobre como guardá-la com segurança”. Houve menor concordância também com os procedimentos na APS, entre os quais destacam-se: "infiltração de substâncias em cavidade sinovial (articulação, bainha tendinosa) (70,8\%)", "biópsia/punção de tumores superficiais de pele com envio de material para análise anatomopatológica" $(73,9 \%)$ e "remoção manual de fecaloma" (74,1\%).

Quando analisamos as contribuições e as alterações realizadas sob a ótica qualitativa, após a consulta pública, destacamos seis pontos que mais chamaram a atenção: (i) abordar o que compete à APS e a necessidade de protocolos com articulação com a rede; (ii) a inclusão de item em que constasse a promoção de práticas integrativas e complementares; (iii) a menção de que, para exames e procedimentos, alguns itens da carteira serão de difícil execução, considerando a infraestrutura de algumas unidades; (iv) a inclusão de itens que meçam a avaliação da fragilidade e avaliação multidimensional em idosos; (v) no texto que antecede a lista seria necessário constar a necessidade da equipe de APS ter suporte de outros serviços da rede de atenção; (vi) a necessidade do reforço e possibilidade de adaptação da carteira à realidade local. 
Quadro 1. Lista de serviços da Carteira de Serviços da Atenção Primária à Saúde do Brasil - CASAPS.

\begin{tabular}{|l|l|}
\hline & \multicolumn{1}{|c|}{ Atenção e Cuidados Centrados no Adulto e no Idoso } \\
\hline 1 & Assistência ao pré-natal da gestante e do parceiro, com utilização da Caderneta da Gestante. \\
\hline 2 & Assistência no período do puerpério. \\
\hline 3 & Assistência à mulher no período do climatério. \\
\hline 4 & $\begin{array}{l}\text { Abordagem em saúde sexual e reprodutiva: orientação individual e em grupos de métodos contraceptivos; } \\
\text { gestão de risco; oferta e dispensação de insumos de prevenção e testes rápidos; disfunçóes sexuais; } \\
\text { avaliação pré-concepção; diagnóstico precoce de gravidez e orientação sobre infertilidade conjugal. }\end{array}$ \\
\hline 5 & Manejo de problemas ginecológicos mais comuns: sangramento uterino anormal, miomatose,
\end{tabular}

corrimento vaginal (abordagem sindrômica) e dor pélvica.

6 Prevenção, identificação, acolhimento e acompanhamento de situações de violência contra mulheres, violência sexual, intrafamiliar e de gênero, preferencialmente em parceria intersetorial com serviços de assistência social e segurança pública.

7 Promoção do aleitamento materno e manejo de problemas mamários comuns relacionados à lactação.

8 Rastreamento para neoplasia de colo uterino e acompanhamento da mulher com diagnóstico de câncer do colo uterino.

9 Rastreamento para neoplasia de mama e acompanhamento da pessoa com diagnóstico de câncer de mama.

10 Acompanhamento de adultos e idosos em cuidados paliativos, com orientação aos familiares/cuidadores.

11 Atendimento das populações em situação de vulnerabilidade, como população de rua, cigana, quilombola, privados de liberdade, dentre outras.

12 Acompanhamento de pessoas com doenças relacionadas ao trabalho.

13 Análise epidemiológica da situação de saúde local.

14 Realizar notificações e ações de vigilância em saúde.

15 Atendimento domiciliar de pessoas em situação de restrição ao leito/acamados, com impossibilidade de locomoção; assim como em situações com indicação de avaliação domiciliar pós óbito, ou de resistência ao tratamento.

16 Atendimento domiciliar para pessoas idosas restritas ao lar ou com dificuldade de mobilidade, incluindo informações, orientações de saúde, aconselhamento e apoio aos familiares/cuidadores.

17 Identificação e acompanhamento da pessoa idosa vulnerável, em risco de declínio funcional ou frágil (multimorbidades, polifarmácia, internações recentes, incontinência esfincteriana, quedas recorrentes, alteração de marcha e equilíbrio, comprometimento cognitivo, comprometimento sensorial, perda de peso não intencional, dificuldade de mastigação e/ou deglutição, sinais e sintomas de transtornos de humor, insuficiência familiar, isolamento social, suspeitas de violência, grau de dependência para atividades da vida diária) com o estabelecimento de um plano de cuidados adaptado a cada caso.

18 Prevenção, identificação, acolhimento e acompanhamento de situações de violência contra idosos, preferencialmente em parceria intersetorial com serviços de assistência social e segurança pública.

19 Prevenção de acidentes domésticos, quedas e fraturas.

20 Promoção do envelhecimento ativo e saudável.

21 Prevenção, identificação, tratamento e acompanhamento de distúrbios nutricionais no adulto e idoso.

22 Rastreamento e aconselhamento para uso abusivo de medicamentos e polifarmácia.

23 Imunização conforme Calendário Vacinal do adulto e do idoso, atentando para situações de surtos de doenças imunopreviníveis e situações clínicas específicas.

24 Identificar e acompanhar adultos e idosos em programas de assistência social ou benefícios sociais.

25 Estímulo a adoção de hábitos de vida saudáveis, como alimentação adequada e saudável, práticas corporais e atividades físicas, controle do álcool, tabaco e outras drogas, dentre outros.

26 Prevenção, busca ativa, diagnóstico, tratamento e acompanhamento da pessoa com hanseníase.

27 Prevenção, rastreamento de sintomáticos respiratórios, diagnóstico, tratamento e acompanhamento da pessoa com Tuberculose (tratamento de primeira linha, tratamento diretamente observado, rastreamento em sintomáticos respiratórios e busca de contatos de pacientes com tuberculose pulmonar).

28 Prevenção, identificação e aconselhamento em relação ao uso abusivo de álcool e outras drogas.

29 Prevenção, identificação, aconselhamento e tratamento em relação ao tabagismo.

30 Prevenção, rastreamento, diagnóstico, tratamento e acompanhamento da pessoa com diagnóstico de Sífilis, Hepatites Virais, outras IST e da pessoa vivendo com HIV, com atenção especial a populações-chave.

31 Promoção da paternidade responsável e ativa.

32 Rastreamento de neoplasia de cólon e reto em pessoas entre 50 e 75 anos. 
Quadro 1. Lista de serviços da Carteira de Serviços da Atenção Primária à Saúde do Brasil - CASAPS.

\begin{tabular}{|c|c|}
\hline & Atenção e Cuidados Centrados no Adulto e no Idoso \\
\hline 33 & Rastreamento e acompanhamento de DM2 em adultos assintomáticos. \\
\hline 34 & $\begin{array}{l}\text { Rastreamento e identificação de Risco Cardiovascular Global (RCVG) baseado em idade, gênero, história } \\
\text { clínica de manifestações cardiovasculares, exame físico focado em manifestações de aterosclerose, medida } \\
\text { da pressão arterial, circunferência abdominal, peso, altura e IMC; para pessoas com } 40 \text { anos ou mais ou } \\
\text { indicadores de risco indeterminados solicitação de colesterol total, hdl colesterol e glicemia de jejum } \\
\text { visando a realização de Escore para Estratificação de RCVG. }\end{array}$ \\
\hline 35 & Identificação, manejo e acompanhamento da pessoa com Diabetes Mellitus (DM). \\
\hline 36 & Identificação, manejo e acompanhamento da pessoa com Hipertensão Arterial Sistêmica (HAS). \\
\hline 37 & $\begin{array}{l}\text { Manejo de doenças cardiovasculares: doença arterial periférica, doença aterosclerótica, dor torácica, } \\
\text { insuficiência cardíaca, palpitações e arritmias crônicas, varizes, sindrome pós-trombótica. }\end{array}$ \\
\hline 38 & $\begin{array}{l}\text { Manejo de condições endocrinológicas mais prevalentes: hipotireoidismo, hipertireoidismo, nódulos de } \\
\text { tireóide, obesidade, dislipidemia. }\end{array}$ \\
\hline 39 & Manejo de pessoas com parasitoses intestinais. \\
\hline 40 & $\begin{array}{l}\text { Manejo de pessoas com exposição e agravos a doenças negligenciadas, como raiva, doença de Chagas, } \\
\text { esquistossomose, leishmaniose (visceral e cutânea), tracoma, malária. }\end{array}$ \\
\hline 41 & Manejo de arboviroses (dengue, zika, febre amarela e chikungunya). \\
\hline 42 & $\begin{array}{l}\text { Manejo das condições mais prevalentes do aparelho digestivo: dispepsia, refluxo gastroesofágico, } \\
\text { alterações de hábito intestinal, gastroenterites, hemorróidas e outras doenças orificiais, dor abdominal, } \\
\text { esteatose hepática, alterações laboratoriais hepáticas. }\end{array}$ \\
\hline 43 & $\begin{array}{l}\text { Manejo de doenças crônicas respiratórias mais prevalentes: Asma, DPOC, tosse crônica, apnéia } \\
\text { obstrutiva do sono, dispnéia, nódulos pulmonares. }\end{array}$ \\
\hline 44 & $\begin{array}{l}\text { Manejo das condições neurológicas mais frequentes: cefaléia, síndromes demenciais, síndrome do túnel } \\
\text { do carpo, epilepsia, acidente vascular encefálico, Doença de Parkinson, vertigem, paralisia facial. }\end{array}$ \\
\hline 45 & $\begin{array}{l}\text { Manejo das condições musculo-esqueléticas mais prevalentes: lombalgia, cervicalgia, osteoartrite, dor } \\
\text { no ombro, bursite/tendinite, dor no quadril, problemas de mãos/punhos, dor no joelho, patologias do } \\
\text { tornozelo e pé, disfunção temporomandibular (DTM), fibromialgia, osteoporose, gota. }\end{array}$ \\
\hline 46 & $\begin{array}{l}\text { Manejo das condições genitourinárias mais prevalentes: doença renal crônica (pacientes renais crônicos } \\
\text { não transplantados), infecção urinária, litíase renal, hiperplasia prostática benigna, incontinência } \\
\text { urinária. }\end{array}$ \\
\hline 47 & $\begin{array}{l}\text { Manejo das condições hematológicas mais prevalentes no âmbito da APS: anemia, anemia falciforme, } \\
\text { linfonodomegalia periférica, leucopenia, leucocitose, trombocitopenia, trombocitose. }\end{array}$ \\
\hline 48 & $\begin{array}{l}\text { Manejo das condições mais prevalentes em dermatologia: acne, celulites, dermatites, erisipela, escabiose, } \\
\text { feridas, furúnculo, herpes simples, herpes zoster, hiperidrose, intertrigo, larva migrans, micoses, miliária, } \\
\text { nevos, pediculose, piodermites, doenças das unhas, psoríase, tungíase, urticária, verrugas. }\end{array}$ \\
\hline 49 & $\begin{array}{l}\text { Manejo das condições mais prevalentes em oftalmologia: conjuntivite, hordéolo e calázio, pterígio e } \\
\text { blefarite. }\end{array}$ \\
\hline 50 & $\begin{array}{l}\text { Manejo das condições mais prevalentes em otorrinolaringologia: vertigem, rinossinusite, otite, } \\
\text { hipoacusia, amigdalite bacteriana, obstrução nasal, apneia do sono, labirintopatia, epistaxe. }\end{array}$ \\
\hline 51 & $\begin{array}{l}\text { Manejo das doenças mais prevalentes em Alergia e Imunologia: rinite alérgica, eczemas alérgicos, } \\
\text { urticária/angioedema, alergia à picada de insetos, alergias alimentares, alergia a medicamentos. }\end{array}$ \\
\hline 52 & $\begin{array}{l}\text { Identificação e manejo das condições psiquiátricas mais prevalentes: sofrimento emocional e tristeza, } \\
\text { transtornos depressivos, de ansiedade, do espectro obsessivo-compulsivo e/ou pós-traumático, } \\
\text { transtornos por uso de substâncias (álcool, tabaco e drogas ilícitas), transtorno de déficit de atenção/ } \\
\text { hiperatividade, transtornos psicóticos e ações de prevenção ao suicídio e automutilação. }\end{array}$ \\
\hline 53 & Oferta de Práticas Integrativas e Complementares no cuidado integral do Adulto e do Idoso. \\
\hline \multirow[t]{2}{*}{54} & Emissão de atestados médicos e laudos, incluindo declaração de óbito, quando solicitados e indicados. \\
\hline & Atenção e Cuidados Centrados na Criança e no Adolescente \\
\hline 55 & $\begin{array}{l}\text { Consulta para acompanhamento do crescimento e desenvolvimento da criança (pesar, medir e avaliar o } \\
\text { desenvolvimento registrando na caderneta da criança). }\end{array}$ \\
\hline 56 & $\begin{array}{l}\text { Promoção e apoio ao aleitamento materno exclusivo até } 6 \text { meses e continuado até } 2 \text { anos ou mais e } \\
\text { manejo de problemas relacionados a lactação. }\end{array}$ \\
\hline 57 & Promoção de alimentação e hábitos saudáveis. \\
\hline 58 & $\begin{array}{l}\text { Aconselhamento para introdução da alimentação complementar na criança, de acordo com as diretrizes } \\
\text { vigentes. }\end{array}$ \\
\hline
\end{tabular}


Quadro 1. Lista de serviços da Carteira de Serviços da Atenção Primária à Saúde do Brasil - CASAPS.

\begin{tabular}{|c|c|}
\hline & Atenção e Cuidados Centrados na Criança e no Adolescente \\
\hline 59 & $\begin{array}{l}\text { Imunização conforme Calendário Vacinal da criança e do adolescente, orientação aos pais ou } \\
\text { responsáveis, avaliação e monitoramento da situação vacinal e busca ativa de faltosos. }\end{array}$ \\
\hline 60 & $\begin{array}{l}\text { Prevenção, identificação, tratamento e acompanhamento de situações relacionadas a distúrbios } \\
\text { nutricionais (baixo peso, desnutrição, sobrepeso e obesidade). }\end{array}$ \\
\hline 61 & $\begin{array}{l}\text { Rastreamento em saúde da criança: triagem neonatal (teste do pezinho e reflexo vermelho) e } \\
\text { identificação precoce de sopros cardíacos. }\end{array}$ \\
\hline 62 & Realizar notificações e ações de vigilância em saúde. \\
\hline
\end{tabular}

63 Análise epidemiológica da situação de saúde local.

64 Vigilância do recém-nascido de risco/vulnerável tendo por base os registros e informes oferecidos pelas maternidades.

65 Suplementação nutricional (vitaminas, minerais, fórmulas) - quando indicado.

66 Prevenção, busca ativa, diagnóstico, tratamento e acompanhamento da criança e adolescente com hanseníase.

67 Prevenção, rastreamento de sintomáticos respiratórios, diagnóstico, tratamento, acompanhamento e investigação de contactantes da criança e adolescente com diagnóstico de tuberculose.

68 Acompanhamento da criança com microcefalia / Zika vírus.

69 Prevenção, rastreamento, diagnóstico, tratamento e acompanhamento da criança e adolescente com sífilis, hepatites virais, HIV e outras ISTs.

70 Prevenção, identificação, aconselhamento e tratamento em relação ao tabagismo.

71 Prevenção, identificação e aconselhamento em relação ao uso abusivo de álcool e outras drogas.

72 Prevenção, identificação, acolhimento e acompanhamento de situações de violência contra crianças e adolescentes, preferencialmente em parceria intersetorial com serviços de assistência social e segurança pública.

73 Prevenção e promoção da segurança da criança e do adolescente contra injúrias físicas: segurança do pedestre, de passageiros de veículos automotores e bicicletas, prevenção de afogamento, queimaduras, quedas, acidentes por armas de fogo e intoxicação.

74 Acompanhamento de crianças e adolescentes com necessidades de cuidados paliativos, oferecendo orientação aos familiares/cuidadores.

75 Atendimento domiciliar de crianças e adolescentes acamadas, com impossibilidade de locomoção, em algumas situações pós óbito, ou de resistência ao tratamento.

76 Identificação e acompanhamento de crianças e adolescentes com dificuldade de aprendizado e problemas relacionados ao contexto escolar e/ou trabalho infantil.

77 Identificação e acompanhamento de crianças e adolescentes com deficiências físicas e/ou mentais.

78 Identificação e manejo de problemas de crescimento e desenvolvimento na infância e adolescência.

79 Identificação e seguimento de crianças e adolescentes em situação de vulnerabilidade (violência, trabalho infantil, medida socioeducativa, acolhimento institucional, em situação de rua, migrantes e refugiados).

80 Atendimento à demanda espontânea com avaliação de risco.

81 Atendimento de urgências/emergências para crianças e adolescentes.

82 Identificação e manejo dos problemas mais comuns do recém-nascido e lactente: cólicas do lactente, regurgitação e vômitos, constipação intestinal, monilíase oral, miliária, dermatite das fraldas, impetigo, dermatite seborréica, icterícia, problemas do umbido, hérnia inguinal e hidrocele, testículo retido, displasia do desenvolvimento do quadril.

83 Identificação e manejo de condições graves de saúde em crianças menores de 2 meses: convulsões, letargia/flacidez, esforço respiratório (tiragem subcostal, batimento de asas do nariz, apnéia), gemido/ estridor/sibilância, cianose central, palidez intensa, distensão abdominal, peso $<2000 \mathrm{~g}$, enchimento capilar lento $(>2 \mathrm{~s})$, pústulas ou vesículas na pele, manifestações de sangramento (equimoses, petéquias e/ ou hemorragias), secreção purulenta do ouvido ou da conjuntiva.

84 Identificação e manejo dos problemas mais comuns na infância: asma, deficiência de ferro e anemia, parasitoses intestinais, febre, infecções de vias aéreas superiores, excesso de peso em crianças, diarréia aguda, rinite alérgica, eczemas alérgicos, urticária/angioedema, alergia à picada de insetos.

85 Atendimento aos agravos prevalentes na adolescência: atraso puberal, puberdade precoce, acne, dor escrotal (criptorquidismo, torção testicular, epididimite, varicocele), ginecomastia puberal, dismenorréia, obesidade, vulvovaginites. 
Quadro 1. Lista de serviços da Carteira de Serviços da Atenção Primária à Saúde do Brasil - CASAPS.

\begin{tabular}{|c|c|}
\hline & Atenção e Cuidados Centrados na Criança e no Adolescente \\
\hline 86 & $\begin{array}{l}\text { Identificação, manejo e acompanhamento do adolescente com Hipertensão Arterial Sistêmica, Diabetes } \\
\text { Mellitus e/ou com dificuldades na acuidade visual. }\end{array}$ \\
\hline 87 & Manejo das doenças crônicas respiratórias mais prevalentes. \\
\hline 88 & $\begin{array}{l}\text { Atendimento e escuta do adolescente em qualquer situação, mesmo desacompanhado, conforme o } \\
\text { Estatuto da Criança e do Adolescente. }\end{array}$ \\
\hline 89 & $\begin{array}{l}\text { Promoção, aconselhamento e cuidado integral à saúde sexual e reprodutiva dos adolescentes: orientação } \\
\text { individual e em grupos de métodos contraceptivos; gestão de risco; oferta e dispensa de insumos de } \\
\text { prevenção e testes rápidos. }\end{array}$ \\
\hline 90 & Identificação, acompanhamento e atenção integral à adolescente gestante. \\
\hline 91 & $\begin{array}{l}\text { Identificação, acompanhamento e manejo de crianças e adolescentes em sofrimento psíquico incluindo } \\
\text { ações de prevenção ao suicídio com adolescentes. }\end{array}$ \\
\hline 92 & Oferta de Práticas Integrativas e Complementares no cuidado integral da Criança e do Adolescente. \\
\hline \multirow[t]{2}{*}{93} & $\begin{array}{l}\text { Identificar e acompanhar crianças e adolescentes inscritos no Programa Bolsa Família ou outros } \\
\text { programas de assitência social ou benefícios sociais. }\end{array}$ \\
\hline & Procedimentos na Atenção Primária à Saúde \\
\hline 94 & $\begin{array}{l}\text { Abordagem e tratamento da pessoa com alterações em seu pé devido a doenças neuropáticas, como } \\
\text { Diabetes Mellitus (DM) e hanseníase. }\end{array}$ \\
\hline 95 & $\begin{array}{l}\text { Administração de medicamentos por via intradérmica, intramuscular, nasal, ocular, oral, parenteral, retal, } \\
\text { subcutânea e tópica. }\end{array}$ \\
\hline 96 & Aferição e monitoramento de Pressão Arterial. \\
\hline 97 & $\begin{array}{l}\text { Aplicação de Ácido Tricloroacético em paciente sem indicação de referenciamento - cauterização química } \\
\text { de pequenas lesões de pele (condilomas, verrugas). }\end{array}$ \\
\hline 98 & $\begin{array}{l}\text { Assistência ao parto em casos de urgência / emergência, quando não houver possibilidade de remoção } \\
\text { para serviços mais adequados em tempo oportuno. }\end{array}$ \\
\hline 99 & Biópsia/punção de tumores superficiais de pele com envio do material para análise anatomopatológica. \\
\hline 100 & Busca ativa de faltosos e contactantes/parcerias de pessoas com doenças transmissíveis. \\
\hline 101 & Cateterismo vesical (de alívio e de demora). \\
\hline 102 & Cirurgia de unha (cantoplastia). \\
\hline 103 & Coleta de exames (urina, fezes, sangue e escarro). \\
\hline 104 & Coleta de material para realização do Citopatológico Cérvico Uterino. \\
\hline 105 & Colocação de imobilização provisória (bandagens e enfaixamentos). \\
\hline 106 & Cuidados com estomas (digestivos, urinários e traqueais). \\
\hline 107 & Curativos simples ou com coberturas especiais. \\
\hline 108 & Desbridamento autolítico, enzimático ou mecânico, e curativo em lesões por pressão ou ulceração. \\
\hline 109 & Drenagem de abscesso. \\
\hline 110 & Drenagem de hematoma subungueal. \\
\hline 111 & Estesiometria (teste de sensibilidade). \\
\hline 112 & Exérese de calosidades. \\
\hline 113 & Exérese de cistos, lipomas e nevos. \\
\hline 114 & Fundoscopia. \\
\hline 115 & Imunização / Administração de vacinas. \\
\hline 116 & Inalação com espaçador e nebulometro. \\
\hline 117 & Infiltração de substâncias em cavidade sinovial (articulação, bainha tendinosa). \\
\hline 118 & Inserção e retirada de DIU. \\
\hline 119 & $\begin{array}{l}\text { Manobras para diagnóstico e reposição otolítica para manejo da vertigem posicional paroxística benigna } \\
\text { (Dix-Halpicke e Epley). }\end{array}$ \\
\hline 120 & $\begin{array}{l}\text { Oferta de Práticas Integrativas e Complementares (auriculoterapia/acupuntura/agulhamento seco/ } \\
\text { infiltração de anestésico em ponto-gatilho). }\end{array}$ \\
\hline 121 & $\begin{array}{l}\text { Ordenha mamária, considerando sempre que necessário a integração com a Rede de Bancos de Leite } \\
\text { Humano. }\end{array}$ \\
\hline 122 & Oxigenioterapia. \\
\hline 123 & Realização de intradermorreação com derivado dermato proteico (PPD). \\
\hline
\end{tabular}


Quadro 1. Lista de serviços da Carteira de Serviços da Atenção Primária à Saúde do Brasil - CASAPS.

124 Realização de Prova do Laço para avaliação de pessoas com quadro clínico suspeito de dengue.

125 Remoção de cerume de conduto auditivo externo (lavagem otológica).

126 Remoção de molusco contagioso.

127 Remoção manual de fecaloma.

128 Remoção não cirúrgica de vermes e parasitas.

129 Retirada de pontos.

130 Retirada/remoção de corpo estranho: farpas de madeira e metálicas, vidro, corpo estranho subcutâneo, ferimento por prego, remoção de anzol, anel, corpo estranho ocular, em cavidade auditiva, nasal, corpo estranho vaginal, anal, desde que sem perfuração.

131 Sondagem nasogástrica.

132 Suturas de lesões superficiais de pele.

133 Tamponamento da epistaxe.

134 Terapia de reidratação oral.

135 Teste $\mathrm{KOH}$ (teste das aminas positivo ou Whiff test).

136 Teste rápido para gravidez.

137 Teste rápido para Hepatite B.

138 Teste rápido para Hepatite C.

139 Teste rápido para HIV.

140 Teste rápido para sífilis.

141 Tratamento de feridas.

142 Tratamento de miĺase furunculóide.

143 Tratamento/cuidado da pessoa com queimaduras (exceto grande queimado).

144 Utilização de ferramentas de abordagem e orientação familiar: genograma, APGAR familiar, avaliação do ciclo de vida familiar, ECOMAPA, Escala Brasileira de Insegurança Alimentar (EBIA), os modelos FIRO e PRACTICE, identificação da dinâmica e estrutura familiar, inclusão das famílias no tratamento de condições complexas e inquéritos alimentares.

145 Utilização de ferramentas de abordagem e orientação comunitária: espaços para abordagem comunitária na unidade de saúde ou outro ponto da comunidade, grupos, realização de oficinas, elaboração de materiais educativos/informativos, territorialização (conhecendo todos os pontos de apoio da comunidade, sejam da saúde ou não), terapia comunitária e utilização dos dados para realização de vigilância epidemiológica.

146 Utilização de referência e contra-referência como ferramentas para o compartilhamento do cuidado com os demais serviços da rede de atenção, bem como os serviços do Sistema Telessaúde Brasil.

147 Verificação de sinais vitais (temperatura, frequência cardíaca, frequencia respiratória, pressão arterial).

\begin{tabular}{|l|l|}
\hline & \multicolumn{1}{|c|}{ Atenção e Cuidados Relacionados à Saúde Bucal - Odontologia } \\
\hline 148 & Procedimentos de Promoção e Prevenção em Saúde Bucal \\
\hline 149 & Ação coletiva de escovação dental supervisionada. \\
\hline 150 & Aplicação tópica de flúor (individual por sessão). \\
\hline 151 & Atendimento odontológico da gestante. \\
\hline 152 & Atividade educativa / Orientação em grupo na atenção primária. \\
\hline 153 & Consulta/Atendimento domiciliar. \\
\hline 154 & Evidenciação de placa bacteriana. \\
\hline 155 & Exame bucal com finalidade epidemiológica. \\
\hline 156 & Orientação de higiene oral. \\
\hline 157 & Orientação de higienização de próteses dentárias. \\
\hline & Procedimentos Clínicos \\
\hline 158 & Acesso a polpa dentária e medicação (por dente). \\
\hline 159 & Adaptação de prótese dentária. \\
\hline 160 & Aplicação de cariostático. \\
\hline 161 & Aplicação de selante (por dente). \\
\hline 162 & Atendimento de urgência odontológica na APS. \\
\hline 163 & Biópsia de tecidos moles da boca. \\
\hline
\end{tabular}


Quadro 1. Lista de serviços da Carteira de Serviços da Atenção Primária à Saúde do Brasil - CASAPS.

\begin{tabular}{|c|c|}
\hline & Atenção e Cuidados Relacionados à Saúde Bucal - Odontologia \\
\hline & Procedimentos de Promoção e Prevenção em Saúde Bucal \\
\hline 164 & Capeamento pulpar. \\
\hline 165 & Cimentação de Prótese Dentária. \\
\hline 166 & Confecção, instalação e ajuste de placa miorelaxante. \\
\hline 167 & Contenção de dentes por splintagem (imobilização dental). \\
\hline 168 & Coroa provisória. \\
\hline 169 & Curativo de demora com ou sem preparo biomecânico. \\
\hline 170 & Curetagem periapical. \\
\hline 171 & Diagnóstico de distúrbio de Articulação Têmporo-Mandibular (ATM). \\
\hline 172 & Drenagem de abscesso da boca e anexos. \\
\hline 173 & Excisão e sutura de lesão na boca. \\
\hline 174 & Excisão de lesão e/ou sutura de ferimento da pele, anexos e mucosas (boca e anexos). \\
\hline 175 & Excisão de rânula ou fenômeno de retenção salivar. \\
\hline 176 & Exodontia de dente decíduo. \\
\hline 177 & Exodontia de dente permanente. \\
\hline 178 & Exodontia múltipla com alveoloplastia. \\
\hline 179 & Frenectomia. \\
\hline 180 & Gengivectomia. \\
\hline 181 & Instalação de prótese dentária. \\
\hline 182 & Moldagem dento-gengival para construção de prótese dentária. \\
\hline 183 & $\begin{array}{l}\text { Oferta de Práticas Integrativas e Complementares na Saúde Bucal (auriculoterapia, acupuntura, } \\
\text { agulhamento a seco, infiltração de anestésico em ponto-gatilho). }\end{array}$ \\
\hline 184 & Primeira consulta odontológica programática. \\
\hline 185 & Profilaxia e remoção de placa bacteriana. \\
\hline 186 & Pulpotomia dentária. \\
\hline 187 & Radiografia interproximal (bite wing) \\
\hline 188 & Radiografia peri-apical. \\
\hline 189 & Raspagem corono-radicular por sextante. \\
\hline 190 & Raspagem e alisamento subgengivais por sextante. \\
\hline 191 & Raspagem, alisamento e polimento supragengivais por sextante. \\
\hline 192 & Realização de procedimento estético em urgência sentida (ex.: necessidade do trabalho). \\
\hline 193 & Reembasamento e conserto de prótese dentária. \\
\hline 194 & Reimplante dental (Por dente). \\
\hline 195 & Restauração de Dente decíduo posterior. \\
\hline 196 & Restauração de dente decíduo. \\
\hline 197 & Restauração de dente permanente anterior. \\
\hline 198 & Restauração de dente permanente posterior. \\
\hline 199 & Retirada de pontos de cirurgias básicas de pele/anexo e mucosas (boca e anexos). \\
\hline 200 & Selamento provisório de cavidade dentária. \\
\hline 201 & Tratamento cirúrgico de hemorragia buco-dental. \\
\hline 202 & Tratamento de alveolite. \\
\hline 203 & Tratamento de Gengivite Ulcerativa Necrosante Aguda (GUNA). \\
\hline 204 & Tratamento de lesões da mucosa oral. \\
\hline 205 & Tratamento de nevralgias faciais. \\
\hline 206 & Tratamento de pericoronarite. \\
\hline 207 & Tratamento endodôntico de dente decíduo. \\
\hline 208 & Tratamento endodôntico de dente permanente anterior. \\
\hline 209 & Tratamento inicial do dente traumatizado. \\
\hline 210 & Tratamento Restaurador Atraumático (TRA). \\
\hline
\end{tabular}




\section{A reorganização dos itens da carteira}

$\mathrm{Na}$ construção da versão final da carteira foram consideradas as atribuições para os profissionais de nível superior, sendo mantidos os atributos essenciais nos grupos "Criança/Adolescente", "Adulto e Idoso", "Ações/Procedimentos na APS", "Saúde Bucal", considerando, portanto, o processo de trabalho dos médicos, enfermeiros e cirurgiões-dentistas. De um total de 209 ações e procedimentos inicialmente listados na consulta pública, entre inclusões e exclusões, ao final de sua criação, foram definidos 210 itens, incluindo o conjunto de ações previstas para a integração entre atenção primária e vigilância em saúde. Cita-se também a importante contribuição advinda da consulta pública com a alteração e qualificação no texto de 109 itens no total: 44 ítens alterados para "Adulto/Idoso", 27 para "Criança/ Adolescente", 17 para "Procedimentos na APS" e 21 para "Saúde Bucal”. Além disso, foram incluídos o detalhamento de insumos, equipamentos e até as referências técnicas necessárias para a realização de cada ação/procedimento por parte da equipe. Ressaltamos que o modelo da carteira nacional pode ser adaptado à realidade e ao contexto municipal em cada uma das unidades da federação, inclusive considerando a disponibilidade da rede de atenção local.

\section{Discussão}

A carteira de serviços é um documento que visa nortear as ações de saúde e apresenta-se como ordenador da APS nos locais onde foi implantada. Seu conteúdo abrange o conjunto das atividades assistenciais e clínicas, de vigilância em saúde, de promoção e de prevenção oferecidos como serviços que objetivam a melhoria de qualidade do cuidado na APS. Para a atual gestão do Ministério da Saúde e da SAPS, ela faz parte do processo de fortalecimento da clínica da APS, estabelecendo-se como guia para as pessoas que utilizam os serviços de saúde, para os profissionais da APS e dos demais níveis de atenção e cuidado em saúde e para os gestores. $O$ ponto de partida para a elaboração deste documento foi a revisão e a avaliação de carteiras de serviços implantadas em importantes cidades brasileiras. O município do Rio de Janeiro adotou, como uma estratégia de inovação gerencial, a carteira de serviços na APS ainda em $2010^{9}$. De forma semelhante, após este movimento iniciado na capital fluminense, importantes cidades brasileiras seguiram na mesma linha com a elaboração do seu próprio documento municipal. Isso ocorreu em Curitiba, Florianópolis, Natal, Belo Horizonte e, mais recentemente, em Porto Alegre ${ }^{10,11,14-16}$. A iniciativa de elaborar carteiras de serviços da APS em âmbito municipal foi incentivada por algumas secretarias estaduais de saúde no Brasil. Mesmo assim, já passados 9 anos da primeira divulgação do documento no Rio de Janeiro, observa-se que, ao considerarmos todos os 5.570 municípios brasileiros, um número ainda muito restrito e pequeno de cidades dispõe de um documento próprio, não conseguindo oferecer e descrever com transparência para sua população o espectro clínico, de vigilância em saúde e preventivo ofertado por suas equipes de APS/ESF. Nesse sentido, a carteira elaborada pelo Ministério da Saúde, de abrangência nacional, visa auxiliar os gestores municipais na implementação de uma lista de ações e serviços da APS. É um documento que não pretende ser excludente, uma vez que o não mencionamento de um sintoma, sinal, diagnóstico, ação ou cuidado não significa que o mesmo não deva ser realizado na APS. É uma ferramenta que deve ser revisada periodicamente, visto a mudança natural do processo de manutenção da saúde e do de adoecimento.

Está estruturada com ênfase no atributo essencial Integralidade - Abrangência do Cuidado - e dividida em Atenção e Cuidados Centrados no Adulto e no Idoso, na Criança e no Adolescente, Procedimentos na APS e Atenção e Cuidados Relacionados à Saúde Bucal. Entre os problemas que visa resolver podemos citar a concentração de pacientes nos níveis secundário e terciário de cuidados em saúde, que buscam por procedimentos inerentes ao âmbito da APS, como remoção de cerume e tratamento da unha encravada, por exemplo. Mas o desafio do fortalecimento da clínica vai além da realização de procedimentos ou intervenções de maior complexidade no ambiente da APS. Passa também pela realização de acompanhamento e de cuidados resolutivos para problemas clínicos altamente prevalentes e de relativa baixa complexidade, como é o caso da Hipertensão Arterial e do Diabetes Mellitus tipo 2. E isso se torna cada vez mais importante com o passar dos anos e o consequente maior número de idosos em nossa população: cuidar, abordar, manejar e trabalhar com prevenção de doenças crônicas não transmissíveis, em nível individual, familiar e comunitário, é um dos importantes papéis da APS elencados na carteira brasileira. Ao descrever 210 ações e serviços típicos do ambiente da APS, distribuídos pela clínica desta, vigilância 
em saúde, atividades de promoção e prevenção em saúde e de abordagem familiar e comunitária, a carteira de serviços propõe um desafio de qualidade na atenção à saúde, que passa também pelo desafio da formação e qualificação dos escopos de práticas dos profissionais da APS estimulando a capacitação clínica de médicos, enfermeiros e odontólogos e ordenando a necessidade de formação pelos programas de residência em Medicina de Família e Comunidade, Enfermagem de Família e Comunidade e Odontologia na APS.

A APS é ordenadora dos sistemas de saúde, mas para que ela possa desempenhar essa função com competência ainda necessita melhorar sua eficiência ${ }^{17}$. Estudos que se propuseram a investigar a presença e a extensão dos atributos da APS no Brasil indicaram que os serviços possuem baixa orientação para APS, sobretudo quando observados os atributos essenciais ${ }^{18-22}$. Dentre os atributos essenciais, a integralidade ainda parece ser um dos desafios mais consistentes. O baixo desempenho deste pode estar relacionado à incapacidade organizacional dos serviços de APS para a oferta de serviços e à heterogeneidade dos mesmos. Nessa perspectiva, é importante ressaltar que um conjunto de ações estruturadas para fortalecimento da APS é fundamental para se obter ganho de eficiência. Isso passa por uma rede de atenção à saúde organizada e com fluxos estabelecidos, melhoria contínua do acesso, investimentos em residências (medicina de família e comunidade e multiprofissional) e a definição de uma carteira de serviços que atenda as necessidades da população.

Outra importante função gerencial propiciada pela carteira de serviços é a de auxiliar na produção da lista de insumos e na estrutura física necessárias para a execução dos procedimentos e serviços pelos profissionais das equipes de APS. Nesse sentido, a Carteira de Serviços da APS do Brasil, em sua versão completa para profissionais e gestores, apresenta em sua estrutura de descrição de cada serviço a lista dos insumos necessários, assim como um breve trecho de orientações relacionadas ao tópico, seguidas ao final pela sessão "Saiba mais em", ponto em que se referencia o serviço do item em avaliação aos conteúdos clínicos elaborados e presentes nos materiais do Ministério da Saúde (Cadernos de Atenção Básica, Protocolos, etc.).
É importante ressaltar que a carteira de serviços desempenha importante papel de indutor de uma maior oferta de ações e procedimentos pelas equipes de APS. Esta característica foi demonstrada em estudo que identificou a oferta de ações e procedimentos pelas equipes de Saúde da Família, tendo por base a Carteira de Serviços do Município do Rio de Janeiro9 ${ }^{9}$ e que comparou a cidade de Rio de Janeiro com outros locais divididos por estratos populacionais ${ }^{23}$. Este estudo concluiu que a cidade do Rio de Janeiro mostrou melhor desempenho quando comparado à média de municípios de grande porte no que diz respeito à oferta de ações de saúde e serviços.

Ao compararmos a carteira brasileira com a de outros países, principalmente as portuguesa e espanhola ${ }^{24,25}$, vemos que a lista estabelecida para o cenário brasileiro contempla a execução de uma APS forte e abrangente em todos seus aspectos. Seguindo o modelo e o exemplo utilizados principalmente na carteira de serviços da Espanha, poderemos incorporar na carteira brasileira, numa próxima revisão, a descrição e a definição dos indicadores utilizados para monitoramento e avaliação da APS no país, ressaltando desta forma cada vez mais o princípio da transparência nas ações da SAPS.

O mais importante princípio da atual gestão da SAPS é colocar as pessoas em primeiro lugar, isto é, fortalecer o SUS para que seja factualmente um sistema de saúde que coloca as pessoas no centro, se consolidando como um sistema de saúde centrado na pessoa. Esse sistema manifesta-se por uma APS forte e clinicamente resolutivo, com transparência, monitoramento, avaliação e oferecendo autonomia e flexibilidade para o processo de gestão realizado em cada cidade brasileira. A carteira de serviços faz isso ao colocar as pessoas no centro do processo e ao contribuir para o fortalecimento da oferta de cuidados próprios da APS. Da mesma forma oferece transparência ao elencar para toda a sociedade a lista de ações e serviços da APS, contribuindo para o monitoramento, a avaliação e os processos de gestão ao permitir a construção de alguns indicadores de acompanhamento para a implementação dos serviços de saúde listados na carteira. 


\section{Colaboradores}

CRH Cunha trabalhou na concepção, elaboração, metodologia, análise de resultados e na redação final. E Harzheim trabalhou na concepção e na redação final. OL Medeiros trabalhou na concepção, metodologia, análise de resultados e na redação final. OP D'Avila trabalhou na concepção, análise de resultados e na redação final. L Wollmann, LA Faller e C Martins trabalharam na redação final.

\section{Referências}

1. Organização Pan-Americana da Saúde (OPAS). Renovação da Atenção Primária em Saúde nas Américas: documento de posicionamento da Organização Pan-Americana da Saúde/Organização Mundial da Saúde (OPAS/OMS). Washington: OPAS; 2007.

2. Organização Mundial da Saúde (OMS). Relatório Mundial de Saúde 2008. Cuidados de Saúde Primários: agora mais do que nunca. Lisboa: OMS; 2008.

3. Organização Mundial da Saúde (OMS). Declaração de Astana. Genebra: OMS; 2019.

4. Organização Mundial da Saúde (OMS). Declaração de Alma-Ata. Genebra: OMS; 1978.

5. Reis JG, Harzheim E, Nachif MCA, Freitas JC, D'Avila OP, Hauser L, Marins CJ, Pedebos LA, Pinto LF. Criação da Secretaria de Atenção Primária à Saúde e suas implicações para o SUS. Cien Saude Colet 2019; 24(9):3457-3462.

6. Macinko J, Starfield B, Shi L. The contribution of primary care systems to health outcomes within Organization for Economic Cooperation and Development (OECD) countries, 1970-1998. Health Serv Res 2003; 38(3):831-865.

7. Kringos SD, Boerma WGW, Hutchinson A, Saltman RB. Building primary care in a changing Europe. Copenhagen: European Observatory on Health Systems and Policies, World Health Organization (WHO); 2015.

8. Starfield B. Atenção primária: equilíbrio entre necessidades de saúde, serviços e tecnologia. Brasília: UNESCO, Ministério da Saúde (MS); 2002.

9. Rio de Janeiro. Secretaria Municipal de Saúde e Defesa Civil (SMSDC). Guia de Referência Rápida. Carteira de Serviços: Relação de serviços prestados na Atenção Primária à Saúde. Rio de Janeiro: SMSDC; 2011.

10. Curitiba. Secretaria Municipal de Saúde (SMS). Carteira de Serviços - Guia para profissionais de saúde Relação de serviços e condições abordadas na Atenção Primária à Saúde. Curitiba: SMS; 2014.

11. Secretaria Municipal de Saúde de Florianópolis. Carteira de Serviços Atenção Primária à Saúde. Florianópolis: Prefeitura Municipal de Florianópolis; 2014.

12. Brasil. Ministério da Saúde (MS). Manual do instrumento de avaliação da atenção primária à saúde: primary care assessment tool pcatool-Brasil. Brasília: MS; 2010.

13. Brasil. Ministério da Saúde (MS). Carteira de serviços da Atenção Primária à Saúde Brasileira: avaliação por convidados externos e consulta pública. [acessado 2019 Out 29]. Disponível em: http://189.28.128.100/dab/ docs/portaldab/documentos/carteira_servico_da APS_consulta_SAPS.pdf

14. Belo Horizonte. Secretaria Municipal de Saúde (SMS). Carteira Orientadora de Serviços do SUS BH. Relação de serviços prestados na atenção primária à saúde. Belo Horizonte: SMS; 2018.

15. Natal. Secretaria Municipal de Saúde (SMS). Carteira de Serviços da Atenção Básica de Natal. Natal: SMS; 2014.

16. Porto Alegre. Secretaria Municipal de Saúde (SMS). Carteira de Serviços da Atenção Primária à Saúde de Porto Alegre. Porto Alegre: SMS; 2019. 
17. Mendes EV. As redes de atenção à saúde. Brasília: Organização Pan-Americana da Saúde; 2011.

18. Ferrer APS. Avaliação da atenção primária à saúde prestada a crianças e adolescentes na região oeste do município de São Paulo [tese]. São Paulo: Universidade de São Paulo; 2013.

19. Elias PE, Ferreira CW, Alves MCG, Cohn A, Kishima V, Escrivão JA, Gomes A, Bousquat A. Atenção Básica em Saúde: comparação entre PSF e UBS por estrato de exclusão social no município de São Paulo. Cien Saude Colet 2006; 11(3):633-641.

20. Silva AS. Avaliação dos atributos da atenção primária à saúde na estratégia saúde da família em municípios do sul de Minas Gerais [tese]. São Paulo: Universidade de São Paulo; 2014.

21. Van Stralen CJ, Belisário SA, van Stralen TBS, Lima AMD, Massote AW, Oliveira CL. Percepção dos usuários e profissionais de saúde sobre atenção básica: comparação entre unidades com e sem saúde da família na Região Centro-Oeste do Brasil. Cad Saude Publica 2008; 24(Supl. 1):s148-s158.

22. Pinto LP, Harzheim E, Hauser L, D'Avila OP, Gonçalves MR, Travassos P, Pessanha R. A qualidade da Atenção Primária à Saúde na Rocinha - Rio de Janeiro, Brasil, na perspectiva dos cuidadores de crianças e dos usuários adultos. Cien Saude Colet 2017; 22(3):771-781.

23. Salazar BA, Campos MR, Luiza VL. A Carteira de Serviços de Saúde do Município do Rio de Janeiro e as ações em saúde na Atenção Primária no Brasil. Cien Saude Colet 2017; 22(3):783-796.

24. Portugal. Portaria no $1368 / 2007$, de 18 de outubro de 2007. Diário da República 2007; 18 out.

25. España. Ministerio de Sanidad y Politica Social. Cartera de servicios de atención primaria - Desarollo, organización, usos y contenido. Madrid: Gobierno de España; 2010.

Artigo apresentado em 30/10/2019

Aprovado em 17/12/2019

Versão final apresentada em 19/12/2019 